DOI: $10.17234 /$ SRAZ.64.1

UDC: 821.133.1.09 Char, R.

UDC: 821.133.1.09 Bataille, G.

Original scientific paper

Reçu le 10 octobre 2019

Accepté pour la publication le 20 décembre 2019

\title{
Georges Bataille et René Char : l'écriture et la question du monde ${ }^{1}$
}

\author{
Sylvain Roux \\ Université de Poitiers \\ sylvain.roux@univ-poitiers.fr
}

Georges Bataille conçoit l'écriture comme une expérience sacrificielle, qui refuse de s'aliéner aux significations que la société donne aux êtres et aux choses, et qui consiste à témoigner de l'existence véritable comme libérée des contraintes de l'action utile et du projet. Cette conception, Bataille l'a exposée dans un texte adressé à René Char («Lettre à René Char sur les incompatibilités de l'écrivain ») et il a cru la retrouver dans l'œuvre poétique de ce dernier. L'objet de cet article est de s'interroger sur la pertinence de ce rapprochement, car la conception du rôle de l'écrivain apparaît très différente chez les deux auteurs, comme le montre en particulier la question du rapport que l'écriture doit entretenir avec le monde.

Mots-clés : écriture, expérience, immanence, impossible, langage, monde, peinture, poésie, sacré, sacrifice, signification.

La question de l'écriture et de son rapport à la philosophie est une question centrale dans l'œuvre de Georges Bataille. Cette dernière, par les références qu'elle emprunte, par l'élaboration conceptuelle qu'elle propose (à travers, notamment, des notions telles que le sacrifice, la dépense ou la souveraineté) ou par le caractère systématique qu'elle tente d'adopter (que l'on songe par exemple à des titres de recueils comme La somme athéologique), semble souvent suivre un modèle d'écriture philosophique. Mais, dans le même temps, Bataille ne se considère pas exactement comme un philosophe. Car il estime que l'entreprise philosophique n'est pas absolument appropriée à son propre projet. Ainsi écrit-il, dans la conclusion de L'Érotisme :

«La philosophie ne sort pas d'elle-même, elle ne peut sortir du langage. Elle utilise le langage de telle manière que jamais le silence ne lui succède. Si bien que le moment suprême excède nécessairement l'interrogation philosophique $»^{2}$.

1 La réalisation de cet article a bénéficié du soutien accordé par les ministères de l'Europe et des Affaires étrangères (MEAE) et de l'Enseignement supérieur, de la Recherche et de l'Innovation (MESRI).

2 L'Érotisme [1957], CEuvres complètes, X, Paris, Gallimard, 1987, p. 268-269. 
La philosophie peut donc contribuer à poser un problème, elle utilise le langage et les mots afin de le formuler et d'en saisir les enjeux, mais la réponse à ce problème la dépasse et se trouve dans une démarche et une expérience silencieuses qui n'appartiennent plus à la philosophie. Malgré tout, et probablement parce que Bataille reconnaît la nécessité de recourir au langage philosophique pour qu'un problème soit posé, il lui arrive d'accepter pour lui-même le qualificatif de philosophe, tout en prenant d'importantes précautions : «s'il est vrai que je suis avant tout un philosophe je suis un philosophe irrégulier et [...] ma philosophie a peu de choses à voir avec la philosophie universitaire $»^{3}$.

Cette méfiance à l'égard de la philosophie conduit donc Bataille à s'interroger sur la possibilité d'une écriture non philosophique, qui lui permettrait d'exprimer ce qui tient la philosophie en échec. Ajoutons par ailleurs que l'avènement d'une telle écriture fait l'objet d'une expérience particulièrement douloureuse et se heurte sans cesse à sa propre difficulté. C'est la raison pour laquelle l'œuvre de Bataille compte un nombre particulièrement important de textes restés inachevés ou non publiés. Il évoque ce problème dans L'Expérience intérieure :

«À peu près chaque fois, si je tentais d'écrire un livre, la fatigue venait avant la fin. Je devenais étranger lentement au projet que j’avais formé. J'oublie ce qui m'enflammait la veille, changeant d'une heure à l'autre avec une lenteur somnolente. Je m'échappe à moi-même et mon livre m'échappe ; il devient presque entier comme un nom oublié : j'ai la paresse de le chercher, mais l'obscur sentiment de l'oubli m'angoisse $»^{4}$.

Cette difficulté trouve peut-être son explication dans le fait que, pour Bataille, la vie authentique (souveraine) et l'expérience qu'elle doit nous procurer, relèvent de l'impossible en ce qu'elles contredisent toutes les exigences habituelles de la $v_{i}{ }^{5}$. Or, la littérature, poétique notamment, doit pourtant chercher à dire cet impossible (la poésie est « l'évocation par les mots de possibilités inaccessibles », indique Bataille dans L'impossible ${ }^{6}$. Mais on ne saurait manquer de relever le paradoxe de cette situation : comment la littérature pourrait-elle être expression de l'impossible, si l'impossible est justement ce qui échappe à la représentation, dans la mesure même où il relève d'une contradiction ou d'une inadéquation entre des fins incompatibles?

Quelle peut donc être cette écriture qui semble si difficile à mettre en œuvre (une écriture impossible parce qu'écriture de l'impossible) mais qui doit pourtant sans cesse être recherchée? Celle-ci est présentée par Bataille comme une écriture sacrificielle, qui s'oppose à la forme d'écriture qui a prévalu en Occident jusqu'ici. Mais pour expliquer en quoi consiste une telle écriture, Bataille recourt à une vaste théorie anthropologique qui donne les clés de la compréhension des conduites

3 «Conférence à la Société d'Agriculture, Sciences, Belles-Lettres et Arts d'Orléans » [1952], CEuvres complètes, IX, Paris, Gallimard, 1979, p. 325.

4 L'expérience intérieure [1943], CEuvres complètes, V, Paris, Gallimard, 1973, p. 72.

5 L'expérience intérieure, CEuvres complètes, V, op. cit., p. 73.

6 L'impossible [1962], CEuvres complètes, III, Paris, Gallimard, 1971, p. 221. 
humaines les plus importantes et qui permet de comprendre comment se libérer de l'écriture occidentale pour promouvoir, à la fois, une existence authentique et la manière d'écrire qui lui correspond. La thèse de Bataille est en effet que c'est dans l'écriture que peut se produire une expérience nouvelle qui nous ramène à notre vérité d'être humain, qui est aussi la vérité de l'être lui-même. Mais Bataille n'a pas seulement cherché à exposer cette nouvelle conception, il a voulu lui reconnaître des prédécesseurs ou lui associer des « alliés substantiels », selon l'expression de René Char ${ }^{7}$. Et c'est justement dans la poésie de ce dernier qu'il a cru reconnaître une démarche similaire à la sienne. Il y a là pourtant une difficulté. On verra en effet que la recherche du poète poursuit un objectif différent de celui de Georges Bataille et qu'il existe donc une divergence profonde entre les deux auteurs. Mais celle-ci a pour mérite de mettre en lumière un problème fondamental, celui de savoir si l'écriture doit nous détourner du monde ou si elle doit nous permettre de le retrouver.

\section{Qu'est-ce qu'un sacrifice?}

Depuis ses premiers textes, une notion a joué un rôle fondamental dans l'élaboration de la pensée de Bataille, et elle se trouve bien sûr au cœur de sa conception de l'écriture. Il s'agit de la notion de sacrifice. Elle n'a cessé d'être utilisée pour penser les différents phénomènes auxquels Bataille s'est successivement intéressé (la religion, l'érotisme, l'art préhistorique, la peinture, la littérature, etc.). Il faut donc commencer par en saisir le sens. On sait que cette notion a été étudiée par des anthropologues, français notamment ${ }^{8}$, et que leurs recherches ont fortement influencé la réflexion de Bataille. Mais il ne s'est pas contenté de reprendre leurs travaux, il a proposé, à partir d'eux, sa propre conception du sacrifice. Il est possible d'en restituer les aspects principaux à partir de deux textes qui sont très proches par leur période de rédaction ${ }^{9}$.

En premier lieu, Bataille considère que l'usage quotidien que nous faisons des objets, qu'il appelle usage profane, est un usage « servile». Des réalités du monde, nous faisons des " choses ", c'est-à-dire des entités que nous pouvons utiliser à notre guise, que nous séparons de nous-mêmes afin de pouvoir les manipuler. L'homme transforme donc ces réalités pour en faire des choses utiles, il ne les

7 Il s'agit du titre d'une partie de la Recherche de la base et du sommet, recueil publié aux Éditions Gallimard en 1955.

8 On songera, en particulier, à l'article célèbre d'Henri Hubert et de Marcel Mauss, « Essai sur la nature et la fonction du sacrifice » paru dans L'Année sociologique, 2, 1899, p. 29138 (repris dans M. Mauss, CEuvres. 1. Les fonctions sociales du sacré, Paris, Les Éditions de Minuit, 1968, p. 193 à 307).

9 Il s'agit de La Part maudite, (Paris, Éditions de Minuit, 1949, § 7 : « Le sacrifice ou la consumation ») et d'un texte écrit en 1948 mais qui ne sera publié que de manière posthume en 1974 aux Éditions Gallimard: Théorie de la religion (Première partie, chapitre III, $\S 1$ et suivants). Pour ces deux textes, voir CEuvres complètes, VII, Paris, Gallimard, 1976. 
considère pas pour elles-mêmes mais en fonction de l'utilité qu'elles ont pour lui. Une telle transformation suppose par ailleurs de privilégier le domaine de l'action. C'est parce que l'homme veut agir en vue de fins, parce qu'il se projette dans l'avenir pour assurer son existence, qu'il en vient à faire des réalités qui l'entourent des choses manipulables et qu'il peut garder en réserve. En ce sens, on peut dire du monde profane qu'il est entièrement subordonné au principe de l'action.

En second lieu, le sacrifice se définit comme ce qui opère la transformation inverse de celle qui vient d'être décrite : son principe est d'annuler ce que l'usage profane avait institué. En ce sens, le sacrifice rend les choses à leur être premier, c'est-à-dire en fait à leur inutilité. Par le sacrifice, elles ne sont plus des choses. Mais ce rapport est annulé par une destruction : en sacrifiant une victime, le sacrifice nie son caractère utilitaire puisqu'elle ne sert plus à rien du fait même de sa destruction. Elle retrouve alors la "vérité du monde intime ${ }^{10}$. Ce que Bataille nomme intimité est «l'immanence entre l'homme et le monde, entre le sujet et $1^{\prime}$ objet ${ }^{11}$. Dans le sacrifice, la distance qui avait été instaurée entre les êtres par le travail et l'action, se trouve abolie (la victime - animal, esclave, etc. - n'est plus une chose qui sert) : le sacrifiant et le sacrifié, la foule qui assiste au sacrifice, tous communient dans un même retour à l'être comme indistinction. Notons par ailleurs qu'il n'est pas nécessaire que la victime soit réellement détruite, elle peut ne l'être que symboliquement. De même, il n'est pas nécessaire que les communiants se nourrissent effectivement de la victime. Là encore, la consumation peut n'être que symbolique. Ce qui compte est d'opérer ce retour au monde dans lequel les relations entre les êtres sont des relations immédiates, qui ne dépendent plus des fins fixées par une représentation et par le travail (qui introduisent une médiation entre ces êtres, selon laquelle ils se rapportent les uns aux autres).

Enfin, le sacrifice suppose donc un changement radical dans l'attitude de l'homme par rapport au monde. Il arrive souvent que l'homme détruise pour consommer mais cette consommation est utile, elle est subordonnée à une fin. L'homme organise même cette opération selon le principe de l'utilité. Au contraire, dans le sacrifice, la destruction se fait en pure perte : la dépense d'énergie, la perte de l'objet sacrifié, ne servent à rien du point de vue du monde profane (ils sont même, de ce point de vue, un appauvrissement qu'il vaudrait mieux éviter). Bataille peut dire que dans ce cas, la dépense est improductive.

Pour Bataille, cette pratique manifeste une tendance profonde de l'être humain, qui, d'un côté, doit chercher à se conserver mais de l'autre cherche aussi à dépenser sans contrepartie parce qu'il retrouve par là une communauté d'être avec le monde que la première tendance conduit à abolir en introduisant une séparation entre le sujet et l'objet (les autres êtres, les choses) ${ }^{12}$. Ce point est

10 La Part maudite [1949], CEuvres complètes, VII, op. cit., p. 63.

11 Théorie de la religion [1948], CEuvres complètes, VII, op. cit., p. 307.

12 Sur ce point, voir La littérature et le mal [1957], CEuvres complètes, IX, op. cit., p. 219. 
absolument essentiel : pour Bataille, le sacrifice n'a pas de caractère utilitaire. On pourrait toujours, en effet, considérer qu'il vise une fin précise, comme assurer le lien social par exemple, et dans ce cas, plus rien ne le distinguerait des actes utiles du monde profane (où l'action intéressée et non pas désintéressée, règne). Au contraire, le sacrifice n'est motivé que par un besoin profond de l'être humain, il s'agit d'une tendance, que l'homme doit épancher et qui, à ce titre, n'entre pas dans le cercle des actions visant une fin que l'on se représente comme utile ${ }^{13}$.

Or, il existe, selon Bataille, un rapport entre cette conception du sacrifice (destruction comme perte sans finalité, comme dépense pure) et le domaine des productions artistiques (notamment littéraires), comme en témoigne ce texte qui figure parmi les premiers de Georges Bataille :

«Sous leur forme majeure, la littérature et le théâtre, qui constituent la seconde catégorie, provoquent l'angoisse et l'horreur par des représentations symboliques de la perte tragique (déchéance ou mort) [...]. Le terme de poésie, qui s'applique aux formes les moins dégradées, les moins intellectualisées, de l'expression d'un état de perte, peut être considéré comme synonyme de dépense : il signifie, en effet, de la façon la plus précise, création au moyen de la perte. Son sens est donc voisin de celui de sacrifice ${ }^{14}$.

Bataille distingue ici deux catégories dans le domaine des productions artistiques. La première est constituée de l'architecture, de la musique et de la danse, et elle se caractérise par le fait qu'elle " comporte des dépenses réelles » et pas seulement des dépenses symboliques. Celles-ci constituent au contraire l'essentiel de la seconde catégorie. La littérature par exemple permet la représentation d'une perte : un individu est destitué de sa fonction, de sa situation ou bien se trouve lui-même anéanti. La littérature théâtrale, à laquelle Bataille songe ici (la tragédie ancienne notamment), s'apparente donc à la représentation d'un sacrifice mais qui n'est pas le sacrifice rituel de la religion. Ce sacrifice ne consiste pas à immoler une victime choisie au sein d'une communauté (sacrifice qui aboutit au maintien du lien entre les membres de cette communauté et en assure le salut). Il s'agit plutôt d'une perte, qui est provoquée par la transgression réalisée par le héros tragique à l'égard des dieux ou du corps social. En ce sens, le personnage est bien une victime sacrifiée : le sort qui lui est réservé le retire du monde bien ordonné de la cité, du monde des choses où tout a une utilité, pour le ramener à cet état que Bataille nomme, comme on l'a vu, intimité de l'être. Enfin, on notera que la poésie jouit ici d'un statut particulier, puisque, dans le domaine de la création littéraire, elle assume plus spécifiquement la fonction sacrificielle (en tous cas, lorsqu'elle n'a pas pris certaines formes dégradées dont témoigne son histoire). On verra plus loin pourquoi c'est à la poésie que revient cette fonction.

13 Ibid., p. 215-216.

14 «La notion de dépense », CEuvres complètes, I, Paris, Gallimard, 1970, p. 307. L'article a paru initialement dans La critique sociale, ${ }^{\circ}$ 7, janvier 1933, p. 7-15. 


\section{L'antinomie de l'art et du sacrifice}

Mais qu'il y ait un rapport entre le sacrifice et l'art ne signifie pas pour Bataille que les deux activités soient au même niveau c'est-à-dire qu'elles aient même valeur et qu'elles puissent jouer exactement le même rôle. Car ce qui compte pour Bataille est la capacité de l'une et de l'autre à rendre possible l'état de perte, de dépense improductive. Et de ce point de vue, elles ne sont justement pas égales. C'est ce qu'il signale dès les années 1930 (c'est-à-dire à la même période que celle où il insiste sur la proximité de la littérature et du sacrifice) dans un texte programmatique tiré d'un ouvrage collectif intitulé Pour un collège de sociologie ${ }^{15}$. On y trouve d'emblée exposée la thèse essentielle de Bataille, que nous avons déjà rencontrée, exposée ici sous la forme d'une opposition entre le fait d'être un «homme entier » et le fait d'être « une des fonctions de la société humaine ». Ces fonctions sont au nombre de trois : l'homme de science, l'homme d'action, l'homme de la fiction. Or, Bataille se livre à une critique violente de ces trois fonctions puisqu'il estime que les hommes qui les exercent ont pour tâche de mentir aux autres hommes (asservis à la production de biens utiles) en leur faisant oublier ce qu'est l'existence elle-même et en leur faisant croire qu'elle doit se subordonner à l'action. Ainsi, pour l'homme de science, le désir de connaissance et de vérité passe avant le désir de la vie pour elle-même. L'homme de l'art (et plus précisément l'homme de lettres) se livre au même mensonge, même si, en apparence, il paraît détaché des besoins habituels des autres hommes, et semble faire l'apologie d'une vie plus authentique.

«Mais que signifient ces fantômes peints, ces fantômes écrits suscités pour rendre le monde où nous nous éveillons un peu moins indigne d'être hanté par nos existences désœuvrées ? Tout est faux dans les images de la fantaisie [...]. Les serviteurs de l'art peuvent accepter pour ceux qu'ils créent l'existence fugitive des ombres : ils n'en sont pas moins tenus d'entrer euxmêmes vivants dans le royaume du vrai, de l'argent, de la gloire et du rang social. Il leur est donc impossible d'avoir autre chose qu'une vie boiteuse. Ils pensent souvent qu'ils sont possédés par ce qu'ils figurent, mais ce qui n'a pas l'existence vraie ne possède rien : ils ne sont vraiment possédés que par leur carrière $»^{16}$.

Le reproche que Bataille adresse ici à l'homme de lettres est de ne vivre qu'une « existence dissociée » (une « vie boiteuse »). D’un côté, il décrit des personnages libérés des servitudes de l'action, de l'autre il ne peut lui-même assumer une telle existence et doit se subordonner à des entités extérieures (État, pouvoirs de toute sorte) au nom desquelles et en vue desquelles il écrit. L'écriture rentre alors, elle aussi, dans la servitude de l'action. Tel est aussi le mensonge dont l'homme

15 Le texte a été publié en 1938 aux Éditions Gallimard. Il s'agit du manifeste du mouvement formé par Bataille et d'autres auteurs et qui contient trois textes de présentation : le premier est de Bataille lui-même («L'apprenti sorcier»), le second de Michel Leiris, le troisième de Roger Caillois.

16 «L'apprenti sorcier », CEuvres complètes, I, op. cit., p. 526. 
de lettres se rend coupable : il décrit une manière d'être qui n'est pas celle qu'il adopte. Mais cette critique est plus radicale encore qu'il n'y paraît car elle ne semble pas reconnaître de solution à la difficulté qu'elle met à jour :

«Le plus souvent, la destinée humaine ne peut être vécue que dans la fiction. Or l'homme de la fiction souffre de ne pas accomplir lui-même la destinée qu'il décrit; il souffre de n'échapper à la fiction que dans sa carrière. Il tente alors de faire entrer les fantômes qui le hantent dans le monde réel. Mais dès qu'ils appartiennent au monde que l'action rend vrai, dès que l'auteur les lie à quelque vérité particulière, ils perdent le privilège qu'ils avaient d'accomplir l'existence humaine jusqu'au bout : ils ne sont plus que les reflets ennuyeux d'un monde fragmentaire ${ }^{17}$.

Ce que Bataille indique ici, c'est qu'il est impossible pour l'écrivain de vivre pleinement et réellement la vie qu'il décrit. Il ne peut que mesurer l'écart qui sépare la vie désintéressée, dés-œuvrée, qui apparaît dans la fiction et la vie qu'il mène. Pire, même s'il voulait réduire ou supprimer cet écart, en tentant de faire correspondre les descriptions de ses fictions avec la vie réelle, il échouerait car celles-ci apparaîtraient dénuées de sens, incapables de donner l'image complète de ce qu'est la vie. La condamnation de la littérature est ici radicale car elle repose sur une antinomie entre l'art et la vie comme pure dépense : les fictions littéraires sont des représentations de cette vie, et du sacrifice qu'est la vie qui refuse de se lier à l'action, mais elles n'en sont pas l'exercice même. La perte, le sacrifice, n'y sont pas éprouvés pour eux-mêmes et en ce sens, la littérature ne cesse d'être un mensonge $\mathrm{e}^{18}$.

\section{L'écriture occidentale comme signification}

Ainsi, la littérature se montre incapable de réaliser le sacrifice, elle se contente de le représenter, de le jouer symboliquement sans jamais l'éprouver réellement. Mais cette situation n'est pas encore la pire de toutes. Comme on l'a vu précédemment, la littérature occidentale, bien souvent, n'a même pas consisté en une telle représentation. Elle s'est plutôt résignée à emprunter la voie tracée par le langage ordinaire, qui est celle de la parole utile, subordonnée à l'action. Bataille utilise un concept particulier pour désigner cette forme d'écriture qu'a empruntée la littérature occidentale, celui de signification. Ce concept apparaît dans un texte qui est la réponse adressée à une question posée par le poète René Char dans la revue Empédocle en 1950 : «Y a-t-il des incompatibilités? ». Bataille y indique que l'incompatibilité qui lui paraît fondamentale se situe entre la vie qui refuse de se

17 Ibid., p. 527.

18 Dans d'autres textes de la même période, cette idée se trouve exprimée avec tout autant de force. Ainsi, dans «La folie de Nietzsche »(CEuvres complètes, I, op. cit., p. 549), Bataille écrit : « celui qui s'est formé jusqu'à l'extrême dans la méditation de la tragédie devra donc - au lieu de se complaire dans "l'expression symbolique" des forces qui déchirent - apprendre la conséquence à ceux qui lui ressemblent ». 
soumettre aux exigences de l'action et l'action elle-même en tant qu'elle impose son exigence à la vie : ainsi, à la « vie sans mesure » s'oppose « l'action sans mesure » (ou « démesurée »). Dire de la première qu'elle est sans mesure, c'est dire ce qui la caractérise en propre (toute vie véritable est le refus de se voir limitée, réduite aux fins qui lui sont imposées de l'extérieur ; elle est le « désir de ce qui peut être aimé sans mesure $»^{19}$ ), mais dire de la seconde qu'elle est sans mesure, c'est exprimer un regret en signalant ce qu'elle ne devrait pas être : car, en étant sans mesure, l'action empêche alors la vie elle-même d'être sans mesure. Malheureusement, la littérature se trouve dans une situation qui lui rend difficile de pouvoir dépasser cette opposition. Car elle repose sur l'utilisation du langage.

«Or, de cet objet, si j'ai tant de mal à parler, c'est que jamais il n'apparaît même dès l'instant où j'en parle, puisque, comme il semble, le langage "est un moment particulier de l'action et ne se comprend pas en dehors d'elle" (Sartre) $»^{20}$.

On le voit, il existe donc une contradiction fondamentale entre les exigences du langage et ce que Bataille cherche à exprimer, qu'il nomme ici « l'objet » et qui désigne la vie sans mesure. Cela le conduit à parler de la « misère de la littérature » puisque le langage par lequel la littérature doit tenter d'exprimer la vie (qui se situe « par-delà l'activité productive », qui est dépense sans finalité, en pure perte) ne peut que se subordonner à l'activité pratique, à l'activité utile, qui lui est pourtant opposée. Pour exposer cette difficulté, Bataille a recours, comme nous l'indiquions précédemment, à une notion particulière, sur laquelle il attire l'attention puisqu'il précise, dans sa réponse à René Char, que « le langage "signifie" ${ }^{21}$, c'est-à-dire qu'il ne peut exprimer que ce qui reçoit un sens de l'action. Or, s'il est essentiellement signification, la littérature, à l'opposé, devrait être la suspension de toute signification. Pour mieux comprendre ce point, il n'est pas inutile de se référer à un autre texte de Bataille, consacré au peintre Manet. Car la notion de signification n'est pas utilisée seulement dans le cadre d'une réflexion sur la littérature. Il s'agit d'un concept qui permet de penser l'ensemble des activités artistiques au cours de leur histoire.

«Mais c'est précisément à Manet que nous devons attribuer d'abord la naissance de cette peinture sans autre signification que l'art de peindre qu'est la "peinture moderne". [...] C'est de Manet que date le refus de "toute valeur étrangère à la peinture", l'indifférence à la signification du sujet $»^{22}$.

«Manet supprima la signification du sujet. Supprimer le sujet, le détruire, est bien le fait de la peinture moderne, mais il ne s'agit pas exactement d'une

19 «Lettre à René Char sur les incompatibilités de l'écrivain » [1950], CEuvres complètes, XII, Paris, Gallimard, 1988, p. 22.

20 Ibid., p. 22.

21 Ibid.

22 Manet [1955], CEuvres complètes, IX, op. cit., p. 131. L'expression “toute valeur étrangère à la peinture" est empruntée par Bataille à André Malraux dans Saturne. Essai sur Goya, Paris, Gallimard, 1950. 
absence : plus ou moins, chaque tableau garde un sujet, un titre, mais ce sujet, ce titre sont insignifiants, se réduisent au prétexte de la peinture $»^{23}$.

«L'intention d'un glissement où se perd le sens immédiat n'est pas la négligence du sujet, mais autre chose : il en va de même dans le sacrifice, qui altère, qui détruit la victime, qui la tue, sans la négliger. Après tout, le sujet des toiles de Manet est moins détruit que dépassé ; il est moins annulé au profit de la peinture nue qu'il n'est transfiguré dans la nudité de cette peinture $»^{24}$.

Si la peinture moderne naît avec Manet, c'est parce qu'il est celui qui, pour la première fois, a su libérer l'art pictural de toute forme de signification. De quelle manière? Tout d'abord, comme l'indiquent les passages que nous venons de citer, Manet suspend l'ensemble des significations utilitaires que la société bourgeoise donne au monde. Jusqu'ici en effet, le peintre devait se soumettre à des institutions qui déterminaient le choix des sujets et le sens qu'il convenait de leur donner. En conséquence, la peinture devait parler, elle était discours mais ce discours était celui des valeurs établies. C'est la raison pour laquelle, par exemple, Manet reproche à ses modèles de prendre des poses, c'est-à-dire de reproduire ce sens imposé, qui préexiste à l'acte de peindre ${ }^{25}$. Mais cela ne signifie pas que la peinture doive se priver de tout sujet. Avec Manet, les sujets classiques de la peinture demeurent mais ils sont vidés de leur sens : la peinture est une « subversion impersonnelle ${ }^{26}$, elle conserve les sujets mais les vide de leur sens. Ainsi, par exemple, dans L'Exécution de Maximilien (1867), Manet, selon Bataille, peint son sujet de manière « insensible », avec une « apathie profonde ${ }^{27}$, contrairement à Goya qui, dans Le 3 mai, représentait un sujet équivalent mais en exprimant l'horreur qu'il suscite en nous. Ce qui frappe dans la peinture de Manet, c'est donc qu'elle n'exprime plus rien, qu'elle arrache les objets et les êtres au monde des valeurs pour les rendre à leur statut premier, qui est celui de l'indistinction et de l'indifférence. Et c'est pourquoi Bataille peut dire que cette peinture est d'abord sacrifice : elle n'inscrit plus le sujet dans le monde du projet et du travail mais le rend à l'immanence première du monde. Mais, il convient aussi de le noter, cette in-signifiance du sujet n'est pas le résultat, pour Bataille, de la liberté nouvellement revendiquée par les artistes qui se réclament de la conception dite de " l'art pour l'art » apparue au XIX ème siècle car dans celleci, le peintre fait alors de sa propre subjectivité le sujet véritable de son $\operatorname{art}^{28}$. L'affirmation du moi est encore une signification tandis qu'il s'agit plutôt de se perdre soi-même, de faire advenir une peinture impersonnelle et de se rendre soi-même impersonnel.

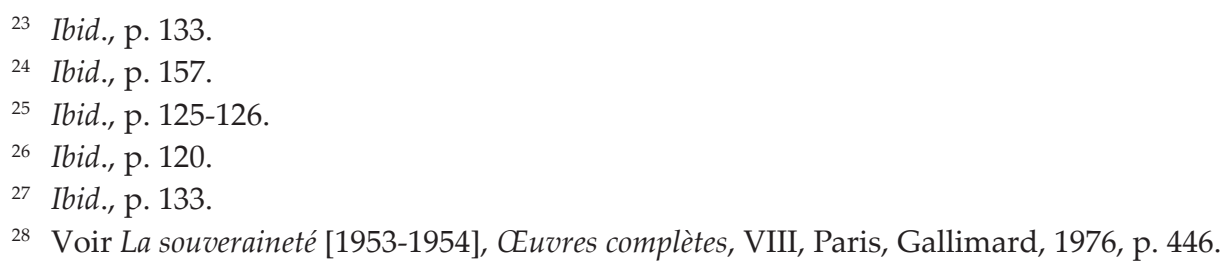


Bataille en tire la conclusion que l'art (la peinture ou la littérature) doit donc s'apparenter à un silence (le « silence des significations $»^{29}$ ) mais ce silence est paradoxal puisqu'il est une manière de parler sans parler, sans se conformer à l'usage traditionnel du langage. Or, la littérature occidentale n'est pas ce silence, puisque la plupart du temps elle a cherché à signifier et continue de se rattacher à la sphère de la signification.

\section{De la possibilité d'une écriture sacrificielle}

Comme l'indiquent déjà nos remarques précédentes, la position de Bataille à l'égard de la littérature et de son lien essentiel avec la notion de sacrifice, a en fait progressivement évolué. Si la littérature peut se faire silence et suspendre toute signification, c'est qu'il ne lui est pas impossible de prendre la forme d'une écriture sacrificielle. Ce tournant, comme l'a montré Koichiro Hamano, dans un ouvrage important consacré à cette question ${ }^{30}$, a eu lieu durant la période de la seconde guerre mondiale, lorsque Bataille rédige plusieurs textes (L'Expérience intérieure, Le Coupable, Sur Nietzsche) qu'il regroupera plus tard sous le titre de La Somme athéologique.

Pour quelles raisons Bataille a-t-il changé d'avis ? La première est qu'il ne conçoit plus les productions sociales des peuples anciens, ou des peuples étudiés par les ethnologues, de la même manière qu'à ses débuts. Cela est particulièrement vrai à propos de la question du potlatch (pratique qui consiste soit à donner des quantités de richesses importantes à un rival dans le but de le défier et de l'obliger, soit à détruire ces richesses dans le même but). Dans «La notion de dépense », il considère que cette pratique peut, certes, entraîner un enrichissement puisqu'elle conduit à multiplier les richesses pour les dilapider, ou entraîner, pour celui qui s'y adonne, un gain sous la forme d'une acquisition de pouvoir ou d'un rang social. Mais ce gain reste secondaire car il est toujours inscrit dans le circuit de la dépense : ce qui est acquis devra être dépensé et s'il s'agit d'un pouvoir acquis, celui-ci est précisément un pouvoir de perdre ( « c'est seulement par la perte que l'honneur et la gloire lui sont liés $\left.{ }^{31}\right)$. Au contraire, dans La Part maudite, Bataille insiste sur « l'ambiguïté et la contradiction » qui sont au cœur de la pratique du potlatch ${ }^{32}$. En effet, cette fois c'est l'acquisition sur laquelle il attire l'attention comme constituant la «fin dernière » du potlatch, mais l'homme qui dilapide en vue de cette acquisition tombe dans une contradiction : il nie «l'emploi servile des biens » en les dépensant mais il le fait en vue d'un usage servile (acquisition de pouvoirs). Bataille va même jusqu'à parler de «mirage », de mystification, à

29 «Lettre à René Char sur les incompatibilités de l'écrivain », CEuvres complètes, XII, op. cit., p. 28.

30 Koichiro Hamano, Georges Bataille. La perte, le don et l'écriture, Dijon, Éditions Universitaires de Dijon, 2004.

31 «La notion de dépense », CEuvres complètes, I, op. cit., p. 311.

32 Pour le raisonnement qui suit, voir La part maudite, CEuvres complètes, VII, op. cit., p. 75-76. 
propos de cette attitude. Mieux, il estime que cette contradiction est à l'œuvre dans toute l'histoire humaine, aussi bien dans les pratiques que dans l'activité de la pensée. D'ailleurs, les deux aspects sont liés : si la contradiction se manifeste dans les pratiques, c'est en fait parce qu'elle repose sur cette activité de la pensée. L'explication en est la suivante : l'intelligence « réduit les objets de pensée à des choses ». Vouloir obtenir un rang par la dépense et la perte, c'est chercher à saisir quelque chose à travers une activité qui demeure en elle-même insaisissable (l'opération de perte, de destruction). C'est l'intelligence qui ne s'accroche qu'à ce qu'elle peut saisir et manipuler et qui introduit au sein de la dépense la possibilité d'un gain. Et Bataille de conclure que le sacrifice n'échappe pas non plus à cette logique :

« c'est que généralement, dans le sacrifice ou le potlatch, dans l'action (dans l'histoire) ou la contemplation (la pensée), ce que nous cherchons est toujours cette ombre - que par définition nous ne saurions saisir $[\ldots] »^{33}$.

Une seconde raison vient expliquer l'évolution de l'analyse de Bataille. C'est qu'il considère désormais que ce qui apparaissait comme la limite de la littérature par rapport au sacrifice réel est en fait l'indice de sa supériorité. Le sacrifice, en tant qu'il est réellement effectué, constitue une perte qui ne peut rester que partielle du point de vue de la société, à moins de menacer purement et simplement son existence. Il lui est donc nécessaire d'en revenir à l'activité utile du monde profane. C'est la tendance à la conservation qui l'emporte donc finalement sur la tendance à la destruction. Mais à l'opposé, l'art, dans la mesure où il n'a pas à se plier aux exigences de la vie utile, peut échapper plus facilement à cette neutralisation de la perte dans l'ordre sensible. Il peut exprimer ce qu'est une perte en échappant au mensonge, à la mystification, du sacrifice réel qui se subordonne en fait au monde profane. Dans l'art, la perte reste symbolique mais non mensongère.

Dans ces conditions, il est donc possible d'opérer un renversement de perspective : le sacrifice n'est pas moins symbolique, en ce sens, que l'activité littéraire qui cherche à figurer la perte et la dépense. Il en reste à une dépense symbolique (même s'il y a un sacrifié) puisqu'il ne menace jamais la communauté sacrifiante, qui n'a rien à perdre de ce sacrifice. Il est donc faux de croire que le passage du sacrifice à sa représentation correspondrait à un passage du réel au symbolique ou qu'il maintiendrait leur opposition : le sacrifice est toujours déjà de nature symbolique.

«Mais la littérature ne fait que prolonger le jeu des religions, dont elle est l'héritière essentielle. Elle a surtout reçu le sacrifice en héritage : cette aspiration à perdre, à nous perdre et à contempler la mort en face a trouvé tout d'abord dans le rite du sacrifice la satisfaction que lui donne encore la lecture des romans : ce fut en un sens un roman, un conte illustré de manière sanglante. Un sacrifice n'est pas moins fictif qu'un roman : ce n'est pas une mise à mort réellement dangereuse, ou coupable, ce n'est pas un crime, mais sa représentation, c'est un jeu. C'est dans son principe le récit d'un crime dont

${ }^{33}$ La part maudite, CEuvres complètes, VII, op. cit., p. 76. 
le prêtre et la victime jouent pour l'assistance l'épisode final. Bien entendu, la victime est vraiment tuée, mais elle n'est, le plus souvent, que l'animal - ou l'homme - anonymes, qui jouent le rôle du dieu - dans d'autres cas, du roi que le prêtre est censé tuer : le rite est en rapport avec un mythe dont il est la représentation périodiquement reprise $»^{34}$.

\section{Georges Bataille et René Char : l'écriture sacrificielle et la question du monde}

Si la littérature peut donc bien reprendre à son compte la fonction sacrificielle, il reste à se demander quelle forme elle doit emprunter pour y parvenir. Comment une écriture sacrificielle est-elle possible ? C'est d'abord tout naturellement vers la forme poétique de l'écriture que se tourne Bataille. En effet, la poésie est par nature moins dépendante du récit, puisqu'elle cherche, en général, à exprimer de manière concise des sentiments et des impressions et non à décrire sous une forme romanesque un ensemble de situations et d'évènements. Or, le récit et la forme romanesque s'inscrivent dans le domaine de l'action, ils mettent en scène des personnages soumis à un projet et à la poursuite de fins utiles. Au contraire, la poésie peut se concentrer sur la forme, et faire un sacrifice de mots, les détourner du sens que l'usage habituel leur réserve. Un texte est particulièrement clair sur ce point :

«De la poésie, je dirai maintenant qu'elle est, je crois, le sacrifice où les mots sont victimes. Les mots, nous les utilisons, nous faisons d'eux les instruments d'actes utiles. Nous n'aurions rien d'humain si le langage en nous devait être en entier servile. Nous ne pouvons non plus nous passer des rapports efficaces qu'introduisent les mots entre les hommes et les choses. Mais nous les arrachons à ces rapports dans un délire.

Que des mots comme cheval ou beurre entrent dans un poème, c'est détachés des soucis intéressés. Pour autant de fois que ces mots : beurre, cheval, sont appliqués à des fins pratiques, l'usage qu'en fait la poésie libère la vie humaine de ces fins $»^{35}$.

Ce texte montre que le langage a bien sûr une fonction utilitaire et que celle-ci ne saurait être contestée. Sans les mots, nous ne pourrions agir sur le monde qui nous entoure puisqu'ils désignent ce sur quoi nous cherchons à avoir prise. Ils

34 L'Histoire de l'érotisme [1951], CEuvres complètes, VIII, op. cit., p. 92. Bataille présente un texte très proche de celui-ci dans la version publiée de L'Érotisme : « La littérature se situe en fait à la suite des religions, dont elle est l'héritière. Le sacrifice est un roman, c'est un conte, illustré de manière sanglante. Ou plutôt, c'est, à l'état rudimentaire, une représentation théâtrale, un drame réduit à l'épisode final, où la victime animale ou humaine, joue seule, mais joue jusqu'à la mort. Le rite est bien la représentation, reprise à date fixe, d'un mythe, c'est-à-dire essentiellement de la mort d'un dieu » (CEuvres complètes, $\mathrm{X}$, op. cit., p. 89).

35 L'expérience intérieure, CEuvres complètes, V, op. cit., p. 156-157. 
nous permettent ainsi de viser des fins et de les réaliser plus facilement. Mais par là même, les mots deviennent prisonniers de notre volonté de projet. Il convient donc de les libérer de cet usage servile et profane. Tel est le sacrifice de la poésie, qui consiste à détacher les mots de toute utilité, et qui leur permet alors de se rapporter aux réalités extérieures, pour elles-mêmes, hors de tout intérêt de notre part. Ainsi, par exemple, des mots comme cheval et beurre perdent leur sens habituel (ils sont sacrifiés, ils meurent à leur sens) en entrant dans de nouveaux rapports auparavant inconnus, notamment grâce aux images, qui rapprochent ce qui ne devrait pas l'être et suggèrent autre chose que ce qui était nommé. Mais cette libération des mots rend possible notre propre libération puisqu'elle nous libère en même temps de notre propre tendance au projet et à l'action, de notre propre aliénation. Bataille reconnaît cependant que la poésie ne remplit cette tâche qu'à la condition d'échapper elle-même à l'aliénation du projet et de la signification : la littérature n'est « rien si elle n'est poésie », mais c'est à la condition que le «langage littéraire » soit « la perversion du langage $»^{36}$.

On le voit, le sacrifice des mots (leur « holocauste $»^{37}$ ), ne consiste pas en un simple jeu formel. Il doit permettre de faire retour aux êtres et à l'être même, en deçà des valeurs que l'action utile leur accorde. Mais comment faut-il entendre ce retour?

"Ce monde en effet n'est pas réductible aux choses, qui nous sont en même temps étrangères et asservies. Ce monde n'est pas le monde profane, prosaïque et sans séduction, du travail (c'est aux yeux des "introvertis", qui ne retrouvent pas dans l'extériorité la poésie, que la vérité du monde se réduit à celle de la chose) : la poésie, qui nie et détruit la limite des choses, a seule la vertu de nous rendre à son absence de limite ; le monde, en un mot, nous est donné quand l'image que nous en avons est sacrée, car tout ce qui est sacré est poétique, tout ce qui est poétique est sacré » ${ }^{38}$.

Le monde qu'il convient de retrouver n'est pas le monde habitable que l'homme s'est construit, et qui est celui de la séparation (une séparation qui s'établit entre les choses mais aussi entre les êtres). Cette séparation permet à l'homme de vivre parce qu'elle lui permet de s'approprier des biens, de les réserver, et de se conserver grâce à eux. Dès lors, elle a tendance à l'enfermer en lui-même au lieu de l'ouvrir aux autres. Au contraire, le monde restitué par la poésie est un monde sans limites, ce n'est plus un ensemble de choses séparées et juxtaposées, mais un monde où l'intimité des êtres les uns avec les autres est alors retrouvée parce que leurs relations ne sont plus que les relations immanentes au sein de la nature. Mais l'importance que Bataille accorde à ce retour à l'immanence, à ce retour au monde, l'amène à rechercher chez d'autres auteurs, en particulier chez certains de ses contemporains, une démarche similaire à la sienne, ou proche de la sienne. Il pense par exemple la retrouver dans la poésie de René Char, qu'il

\footnotetext{
36 Ibid., p. 173.

37 Ibid., p. 169.

38 La Littérature et le Mal [1957], CEuvres complètes, IX, op. cit., p. 226.
} 


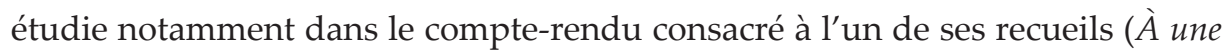
sérénité crispée $)^{39}$. Ce rapprochement peut se justifier par la proximité évidente que manifestent leurs conceptions de la poésie : dans les deux cas, celle-ci est présentée comme essentielle parce qu'elle est placée au-dessus de toutes les autres activités humaines. Le poète est cet être souverain qui échappe aux exigences de la vie commune soumise à l'action utile ${ }^{40}$. Cette proximité se manifeste d'ailleurs par un vocabulaire commun. Ainsi, le terme de "souveraineté » apparaît chez les deux auteurs ${ }^{41}$ et René Char évoque même, dans le recueil auquel s'intéresse Bataille, « l'extase devant le vide » ${ }^{42}$, formule qui apparaît très proche de celles qu'utilise Bataille de son côté, notamment dans L'Expérience intérieure.

Mais il n'est pas sûr que les mots aient ici le même sens. Lorsque René Char écrit :

«Si l'homme parfois ne fermait pas souverainement les yeux, il finirait par ne plus voir ce qui vaut d'être regardé $»^{43}$,

il n'indique pas que la poésie serait une manière de revenir à l'intimité de l'être comme ensemble de relations immanentes entre les êtres, mais qu'elle est la possibilité de découvrir par-delà les apparences communes, le sens véritable des choses ${ }^{44}$. Le poète ne veut pas ramener les choses à leur in-signifiance mais retrouver le sens qui nous est demeuré caché. D’ailleurs, dans son recueil, René Char n'évoque-t-il pas

39 «René Char et la force de la poésie », Critique, n 53, octobre 1951, p. 819-828. Voir CEuvres complètes, XII, op. cit., p. 126-130. Sur la relation entre Georges Bataille et René Char, on pourra consulter l'article de Jean-Luc Steinmetz, «Souveraineté de la poésie : René Char et Georges Bataille », Revue La Licorne, $n^{\circ}$ 13, 2014 (en ligne : http://licorne. edel.univ-poitiers.fr/index.php?id=5871).

40 On se reportera par exemple au début du recueil, qui présente une opposition entre la poésie d'une part, le travail et l'utilité d'autre part, qui n'est pas sans rappeler celle proposée par Bataille : «Produire (travailler) selon les lois de l'utilité, mais que cet utile ne serve à travers tous qu'à la personne de la poésie [...] » (CEuvres complètes, Paris, Gallimard (Bibliothèque de la Pléiade), 1983, p. 751).

41 «La souveraineté obtenue par l'absence dans chacun de nous d'un drame personnel, voilà le leurre » (ibid., p. 752); «Au centre de la poésie, un contradicteur t'attend. C'est ton souverain. Lutte loyalement contre lui » (ibid., p. 754) ; «Peu d'états souverains m'apparaissent comme un point culminant [...]» (ibid., p. 759).

42 «Après l'ultime distorsion, nous sommes parvenus sur la crête de la connaissance. Voici la minute du considérable danger : l'extase devant le vide, l'extase neuve devant le vide frais » (ibid., p. 753).

43 Feuillets d'Hypnos, 59 : voir CEuvres complètes, op. cit., p. 189. Cet aphorisme est cité par Bataille au début de Méthode de méditation [1947], CEuvres complètes, V, op. cit., p. 192.

44 Les autres occurrences du terme souveraineté que l'on rencontre dans le recueil de René Char (voir note 41) montrent qu'il n'est pas nécessairement employé pour désigner le poète lui-même et son attitude (ainsi que sa fonction) mais pour indiquer ce à quoi il se trouve confronté et à quoi il doit s'opposer. Ainsi : «Au centre de la poésie, un contradicteur t'attend. C'est ton souverain. Lutte loyalement contre lui ». Et si une forme de souveraineté saisit le poète lui-même, ce n'est pas de manière positive. 
« cet instant où la beauté, après s'être longtemps fait attendre surgit des choses communes, traverse notre champ radieux, lie tout ce qui peut être lié, allume tout ce qui doit être allumé de notre gerbe de ténèbres $»^{45}$.

Il existe donc une beauté, au-delà de l'apparence commune des choses et que les hommes ne savent plus voir mais que le poète apprend à voir ${ }^{46}$. Le poète est ce voyant, qui a pour mission de guider les hommes vers cette part secrète où tout se réconcilie, de nous ramener vers l'ordre du monde :

«Dans le tissu du poème doit se retrouver un nombre égal de tunnels dérobés, de chambres d'harmonie, en même temps que d'éléments futurs, de havres au soleil, de pistes captieuses et d'existants s'entr'appelant. Le poète est le passeur de tout cela qui forme un ordre. Et un ordre insurgé $»^{47}$.

L'entreprise poétique exprime des chemins inconnus ( "tunnels dérobés », « pistes captieuses »), des moments de bonheur (" chambres d'harmonie », « havres au soleil ») ou de partage (« existants s'entr'appelant»), mais ces différents éléments s'organisent entre eux en un ensemble que le poète n'hésite pas à appeler un « ordre ». Et la tâche de ce dernier est de rappeler cet ordre qui est l'ordre même des choses (il est un « passeur »). Mais cet ordre est en même temps un « ordre insurgé » contre un autre ordre, factice et trompeur, instauré par les hommes. Ce dernier, pourtant, menace toujours de l'emporter et d'effacer l'ordre réel, il faut donc sans cesse œuvrer à rappeler aux hommes ce qu'est l'ordre des choses :

«Le monde jusqu'ici toujours racheté va-t-il être mis à mort devant nous, contre nous ? Criminels sont ceux qui arrêtent le temps dans l'homme pour $l^{\prime}$ hypnotiser, pour perforer son âme $»^{48}$.

Le sens de ce rapport au monde, si différent semble-t-il, de celui qu'évoque Bataille, c'est peut-être Albert Camus qui a su le percevoir avec la plus grande lucidité. Dans ses Cahiers, il écrit :

« Petite baie avant Tenès, au pied des Chaînes montagneuses. Demicercle parfait. Dans le soir tombant, une plénitude angoissée plane sur les eaux silencieuses. On comprend alors que, si les Grecs ont formé l'idée du

45 CEuvres complètes, op. cit., p. 757.

46 Sur ce point, voir les analyses de Georges Mounin, Avez-vous lu Char ? [1947], Paris, Gallimard (Folio-Essais), 1989, p. 101-103 (« pour le poète il s'agit non de dépayser mais de rapatrier », p. 103) et p. 145 sq. (à propos de la conception du poète comme mage et de sa fonction prophétique).

47 À une sérénité crispée, CEuvres complètes, op. cit., p. 760.

${ }^{48}$ Ibid., p. 760. Qu'il y ait un ordre ou qu'il y ait un monde, ne signifie pas pour autant que ceux-ci soient pure harmonie pour René Char. Comme Éric Marty l'a bien montré, ce dernier n'est pas « une sorte de chantre provençal d'un paysage perdu et regretté » (René Char, Paris, Éditions du Seuil, 1990, p. 36). Pour lui, en particulier dans Le marteau sans maître, la nature apparaît comme violente et anarchique, puissante et pas simplement comme «banalement heureuse, aromatique et ensoleillée » (ibid., p. 167). Mais cette violence est justement l'ordre qui lui est propre et par lequel elle peut résister à la tentative d'asservissement que l'homme lui oppose. 
désespoir et de la tragédie, c'est toujours à travers la beauté et ce qu'elle a d'oppressant. C'est une tragédie qui culmine. Au lieu que l'esprit moderne a fait son désespoir à partir de la laideur et du médiocre.

Ce que Char veut dire sans doute. Pour les Grecs, la beauté est au départ. Pour un européen, elle est un but, rarement atteint. Je ne suis pas moderne $»^{49}$.

Bataille ne conçoit donc probablement pas de la même manière que René Char la finalité de l'acte poétique. Pour lui, le retour à l'immanence de la nature n'est point le retour à une nature harmonieuse, à un ordre caché en lequel nous devrions trouver notre mesure. La poésie doit au contraire détruire cette volonté de retrouver un autre ordre ou un sens caché, elle doit être l'expression de la perte de soi et du monde (et non un moyen de retrouver le monde) car elle vise une expérience impersonnelle dans laquelle le sujet s'abolit ainsi que l'objet qu'il prétendrait atteindre.

Ces remarques nous amènent donc à formuler notre hypothèse concernant la nature de l'écriture sacrificielle que Bataille veut développer. Celle-ci ne consiste pas en une nouvelle forme d'écriture, qui coexisterait avec d'autres formes plus anciennes. Il s'agit plutôt d'une subversion de toutes ces formes d'écriture, d'une subversion générale (appliquée à toutes ces formes) et méthodiquement conduite. Il est possible de le vérifier en faisant retour aux différents " projets » d'écriture de Bataille. En premier lieu, l'écriture des textes théoriques apparaît bien comme une subversion de l'écriture philosophique. Nous avons indiqué dans nos remarques introductives, que Bataille cherchait à présenter sa pensée sous une forme systématique, en empruntant une démarche conceptuelle particulièrement prononcée. Mais il est frappant de constater que cette présentation philosophique en détourne complètement le sens. La philosophie est tout entière tournée vers l'acquisition d'un savoir, elle distingue des objets qu'elle essaye de connaître en les séparant les uns des autres. Au contraire, l'expérience que revendique Bataille vise le non-savoir : lorsque nous sortons de tout projet, lorsque nous cessons de nous soumettre aux exigences de l'action, et que toute dépense devient improductive, c'est l'être comme perte que nous éprouvons et cette perte ne nous donne rien, ne nous fait rien posséder ni garder (comme un savoir en réserve) ${ }^{50}$. En second lieu, l'écriture de Bataille prend la forme d'une subversion de l'écriture spirituelle (de tradition chrétienne en particulier). Celle-ci vise à rendre compte de l'expérience mystique qui prétend dépasser l'entendement pour accéder à une union directe avec Dieu. Bataille maintient le même mouvement de dépassement des opérations de l'entendement, la même volonté d'accéder à l'absolu par des moyens qui

49 Albert Camus, Carnets : Cahier V. Septembre 1945 - Avril 1948. Voir CEuvres complètes, II, Paris, Gallimard («Bibliothèque de la Pléiade »), 2006, p. 1111. Sur l'appréciation par Albert Camus de l'œuvre de René Char, voir aussi, dans le même volume (p. 764-768) : «Ce soir le rideau se lève sur... René Char» (1948).

50 Sur ce point, voir, par exemple, L'Expérience intérieure, CEuvres complètes, V, op. cit., p. 15. 
consistent en un retour à soi (l'expérience doit être intérieure), mais il les subordonne à une dissolution du rapport entre sujet et objet qui ne ferait apparaître aucune réalité supérieure mais seulement le vide, le néant de l'être. Ainsi, il n'hésite pas à rebaptiser cette expérience intérieure d'un nom emprunté aux traditions spirituelles : " précédemment, je désignais l'opération souveraine sous les noms d'expérience intérieure ou d'extrême du possible. Je la désigne aussi maintenant sous le nom : méditation $»^{51}$. Enfin, comme nous l'avons vu, Bataille accorde une importance particulière à la poésie puisqu'elle constitue, pour lui, l'essence même de toute activité littéraire authentique. Mais il condamne la poésie soumise au projet, à l'entreprise de la signification et lui préfère la poésie qui exprime la perte et la dépense improductive. En ce sens, au lieu de nous restituer un monde, de nous rendre au monde, elle détruit tout sentiment d'appartenance à un monde (conçu comme belle totalité ordonnée). Car le monde est toujours un monde de valeurs, rendu possible par le travail. La poésie ne peut donc que détruire toute idée de monde au profit d'une expérience impersonnelle de l'être. Bataille opère donc une troisième subversion, celle de l'écriture poétique. Sur ce point, il n'est pas certain que l'œuvre de René Char, comme nous l'avons vu, s'accorde avec un tel projet : si elle dénonce le monde artificiel de l'utilité, c'est pour mieux reconduire, en-deçà de celui-ci, au monde lui-même, enfin compris en son sens véritable ${ }^{52}$.

\section{Bibliographie :}

Bataille Georges, CEuvres complètes, 12 volumes, Paris, Gallimard, 1970-1988.

Camus Albert, CEuvres complètes, II, Paris, Gallimard coll. "Bibliothèque de la Pléiade », 2006. En particulier : Carnets : Cahier V. Septembre 1945 -Avril 1948.

Char René, OEuvres complètes, Paris, Gallimard, coll. «Bibliothèque de la Pléiade », 1983.

Didi-Huberman Georges, La Ressemblance informe ou le gai savoir visuel selon Georges Bataille, Paris, Macula, 1995.

Hamano Koichiro, Georges Bataille. La perte, le don et l'écriture, Dijon, Éditions Universitaires de Dijon, 2004.

51 Méthode de méditation, CEuvres complètes, V, op. cit., p.219.

52 Ces remarques sont aussi de nature à mieux faire comprendre les difficultés évoquées en introduction : si, pour Bataille, l'écriture fait l'objet d'une difficulté particulière, ce n'est évidemment pas pour des raisons qui tiennent à une dimension personnelle. Dans la mesure où l'écriture est subversion de toutes les autres formes d'écriture, où elle vise la perte et l'expression de la perte, elle s'oppose donc à tout projet (et l'écriture d'un livre ne relève-t-elle pas encore d'un projet ?), à la dimension de l'action utile et suscite par là même sa propre difficulté, voire sa propre impossibilité. Si les œuvres de Bataille sont souvent inachevées, c'est que l'inachèvement est constitutif de l'acte même d'écrire, s'il veut rester fidèle à sa vocation (qui est de nous conduire à l'état d'extase qui découle de l'expérience intérieure de la dépossession du sens et du projet) et c'est pourquoi la subversion est peut-être, pour Bataille, une des seules manières de pouvoir encore écrire. 
Hollier Denis, Les Dépossédés (Bataille, Caillois, Leiris, Malraux, Sartre), Paris, Les Éditions de Minuit, 1993.

Marmande Francis, Georges Bataille politique, Lyon, Presses Universitaires de Lyon, 1985.

Marmande Francis, Le pur bonheur, Georges Bataille, Paris, Lignes, 2011.

Marty Éric, René Char, Paris, Éditions du Seuil, 1990.

Mong-Hy Cédric, Bataille cosmique. Du système de la nature à la nature de la culture, Paris, Lignes, 2012.

Mounin Georges, Avez-vous lu Char ? [1947], Paris, Gallimard, coll. « Folio-Essais », 1989.

Sichère Bernard, Pour Bataille. Être, chance, souveraineté, Paris, Gallimard, coll. « NRF, L'Infini », 2006.

Surya Michel, Georges Bataille, la mort à l'œuvre [1987], Paris, Gallimard, coll. « Tel », $2012^{3}$.

Veyne Paul, René Char en ses poèmes, Paris, Gallimard, coll. « Essais », 1990.

Wang Chunming, Liberté et souveraineté : le problème de l'expérience chez Sartre et Bataille. Thèse de doctorat en Philosophie soutenue à l'Université Charles de Gaulle - Lille III en décembre 2015.

\section{Georges Bataille i René Char: pismo i pitanje svijeta}

Georges Bataille shvaća pisanje kao žrtveno iskustvo, što se odbija otuđiti u značenjima koja društvo daje bićima i stvarima, iskustvo koje se sastoji u svjedočenju o istinskoj egzistenciji, slobodnoj od prisila korisnog djelovanja i projekta. Tu je koncepciju Bataille izložio u tekstu upućenom Renéu Charu (»Pismo Renéu Charu o inkompatibilnostima pisca«) ; smatrao je da je pronalazi u Charovom pjesničkom djelu. Predmet ovog članka je ispitivanje pertinentnosti tog približavanja, jer su koncepcije uloge pisca vrlo različite u ta dva autora, kao što to navlastito pokazuje pitanje odnosa koje pisanje mora imati sa svijetom.

Ključne riječi: pisanje, iskustvo, imanencija, nemoguće, jezik, svijet, slikarstvo, pjesništvo, sveto, žrtva, značenje 